\title{
Elektroporation
}

\section{Einsatz von Elektroimpulsen für die Biotechnologie von Mikroalgen}

WOLFGANG FREY ${ }^{1}$, PETER NICK ${ }^{2}$

${ }^{1}$ ARBEITSGRUPPE BIOELEKTRIK, INSTITUT FÜR HOCHLEISTUNGSIMPULS- UND MIKROWELLENTECHNIK, KARLSRUHER INSTITUT FÜR TECHNOLOGIE

2 ABTEILUNG MOLEKULARE ZELLBIOLOGIE, BOTANISCHES INSTITUT, KARLSRUHER INSTITUT FÜR TECHNOLOGIE

\section{Electroporation is used to extract cells without the need for solvents.} However, electrical fields can also be used to deploy biological signalling. Using microalgae as paradigm, we show the potential of electro-modulation for biotechnology. In Haematococcus, metabolic genes astaxanthin synthesis can be triggered. Likewise, electrical triggering of programmed cell death support energy-efficient, mild, and clean purification of Chlorella proteins for applications in food industry.

DOI: $10.1007 / \mathrm{s} 12268-022-1688-7$

(C) Die Autoren 2022

Die Elektroimpulsbehandlung wird seit mehr als 50 Jahren zur Öffnung der Plasmamembran lebender Zellen angewandt. Das biologische Material befindet sich dazu in wässriger Suspension zwischen zwei Elektroden, an welche kurze Hochspannungsim- pulse mit einer Dauer von Mikrosekunden bis zu einigen Millisekunden angelegt werden. Das in der Suspension erzeugte elektrische Feld lädt die Plasmamembran der Zellen auf. Die Spannung über die Membran steigt immer mehr an, weit über das physiologisch übliche Maß, was zu einer Reorientierung der Phospholipidmoleküle der Membran in Form von wassergängigen Poren führt [1]. Die Plasmamembran, die bisher den Stoffaustausch zwischen Zellinnerem und Umgebung streng kontrolliert hat, verliert ihre Integrität, sodass nun Moleküle in die Zelle hineinoder aus der Zelle hinausgelangen können.

Obwohl noch längst nicht alle Phänomene der Permeabilisierung der Plasmamembran verstanden sind, findet die Elektroimpulsbehandlung bereits breite Anwendung in der Bioverfahrenstechnik. Zucker kann mit geringerem Energieaufwand aus Zuckerrüben gewonnen werden, eine Weinmaischebehandlung erlaubt geringere Prozesszeiten

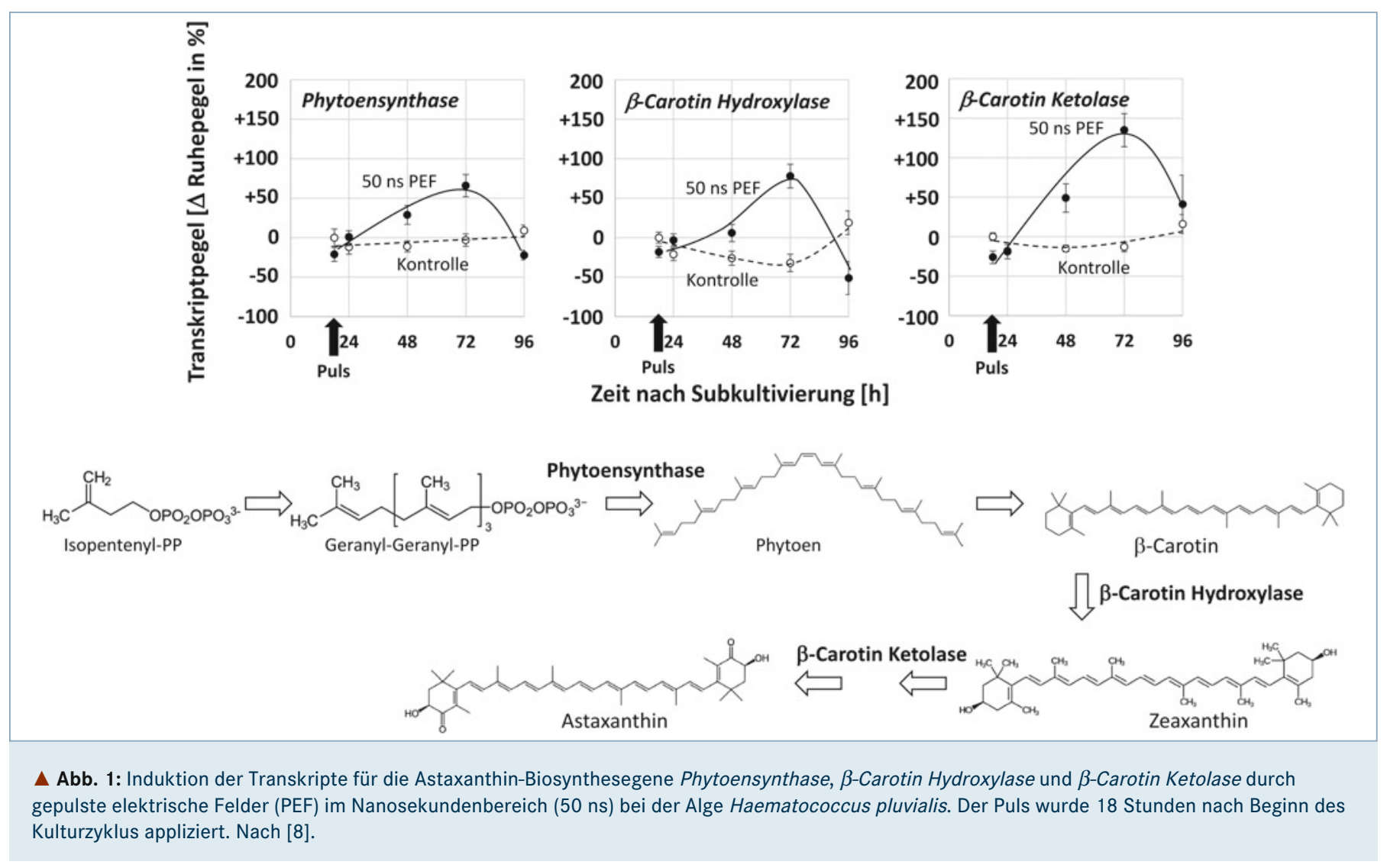


Abb. 2: Arbeitsmodell der Wirkung von nsPEFs auf die Expression von Astaxanthin-Biosynthesegenen. Für kurze Pulsdauern (25 ns) wird die Plasmamembran permeabilisiert, wodurch Calciumionen einströmen und membranständige NADPH-Oxidasen aktiviert werden, sodass reaktive Sauerstoffspezies (ROS) entstehen. Diese Signale führen zu Aktivierung von Biosynthesegenen im Zellkern. Für längere Pulsdauern (50 ns) wird zusätzlich noch die Chloroplastenmembran perforiert. Dies löst zusätzlich ein retrogrades Signal aus, was die Antwort der Genexpression im Zellkern moduliert. Nach [8].
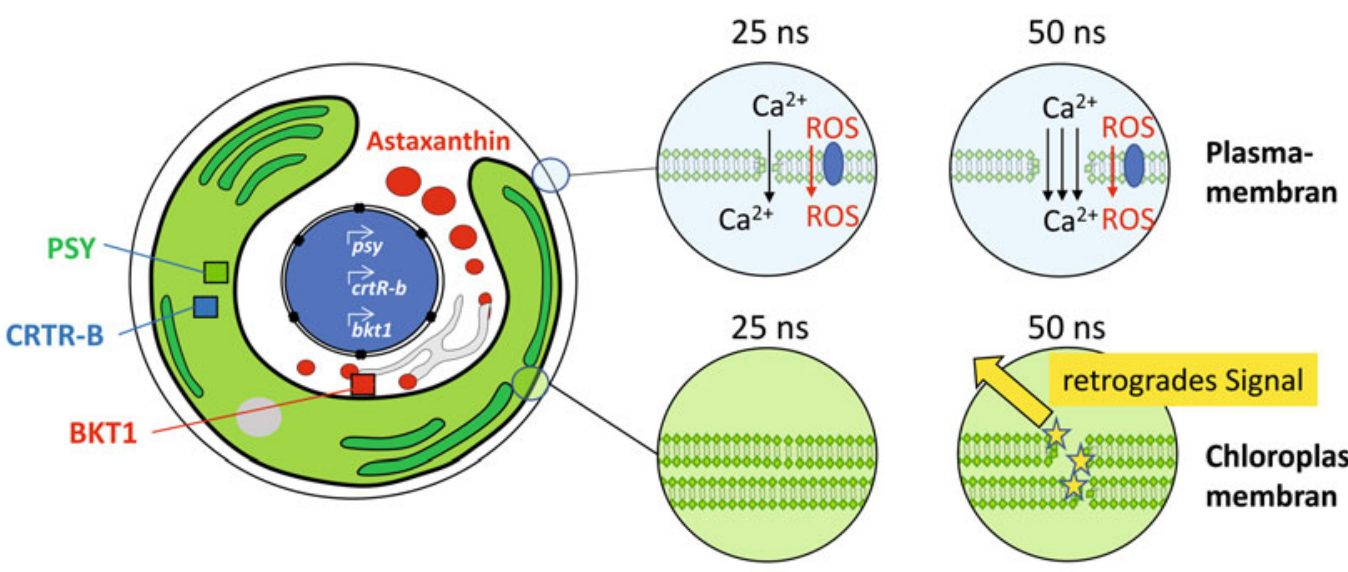

Phytoensynthase

$\beta$-Carotin Hydroxylase

$\beta$-Carotin Ketolase

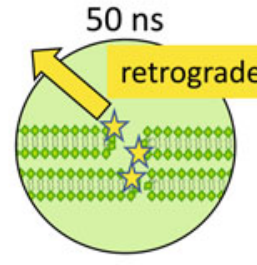

des Signal

Chloroplastenmembran bei der Weinherstellung, die Trocknung biologischer Produkte kann beschleunigt werden und Wasser kann wirkungsvoll entkeimt werden - um nur einige zu nennen [2]. In den letzten Jahren konnte zudem gezeigt werden, dass die Elektroimpulsbehandlung vorteilhaft zur Extraktion von Mikroalgen eingesetzt werden kann [3, 4, 5].

\section{Elektroimpulsbehandlung von Mikroalgen - Potenzial und Herausforderungen}

Mikroalgen produzieren sehr effizient Proteine, Lipide und andere Wertstoffe wie Antioxidantien. Allerdings schützen sie diese nicht nur durch eine Plasmamembran, sondern auch mit einer teils sehr robusten Zellwand, was verfahrenstechnisch einen Zellaufschlussschritt vor der eigentlichen Extraktion erfordert. Dazu hat sich die Elektroimpulsbehandlung als besonders vorteilhaft erwiesen, da sie eine durchlässige Zellbegrenzung schafft, ohne die Zelle zu fragmentieren (Abb. 1). Das erleichtert die Gewinnung von Koppelprodukten. Nach Elektroimpulsbehandlung können beispielsweise in einem Kaskadenprozess zunächst wasserlösliche Proteine gewonnen werden und im nachfolgenden Schritt die intrazellulär verbliebenen Lipide mittels Lösemittelextraktion gewonnen werden [6].

Auch in der Biotechnologie ist Energieeinsparung ein stets dominantes Thema. Derzeit müssen etwa pro Kilogramm Biotrockenmasse etwa ein Megajoule an Energie für den Zellaufschluss mit gepulsten elektrischen Feldern aufgewandt werden. Das ist weniger als für herkömmliche Aufschlussverfahren wie Hochdruckhomogenisation oder Kugelmahlen aufzuwenden ist [5]. Dennoch ist es im Sinne einer nachhaltigen Erzeugung wichtig, diesen Energieeintrag weiter zu reduzieren.

\section{Mehr als nur Porenbildung - biologische Wirkungen von Elektroimpulsbehandlungen}

Die enge Zusammenarbeit von Zellbiologie, Biophysik und Elektrotechnik leistet hierzu einen wichtigen Beitrag. Elektrische Impulse können nämlich weit mehr als Membranen perforieren - sie sind wichtige Elemente von Signalketten, mit denen Zellen auf Änderungen ihrer Umgebung reagieren und sich an widrige Bedingungen anpassen können. Die elektrische Signalleitung unserer Nervenzellen ist nur ein prominentes Beispiel dafür. Auch wenn Nervenzellen sich auf diese Form der Signalleitung spezialisiert haben, um damit schnelle Bewegungen effizient steuern zu können, finden sich elektrisch gesteuerte Signalvorgänge bei allen Lebensformen, auch bei den Mikroalgen. Dies eröffnet die Möglichkeit, mit elektrischen Impulsen biotechnologisch erwünschte Vorgänge auszulösen. Ein Signal muss nicht stark sein, um wirken zu können, es genügt, wenn es präzise ist es geht ja um die Übertragung von Information, nicht um die Übertragung von Energie.

In der Tat konnten wir diese Logik experimentell untermauern. In einem ersten Projekt mit der einzelligen Grünalge Chlamydomonas reinhardtii konnten wir mit sehr kurzen (im Nanosekundenbereich), aber intensiven Pulsen die Zellen dazu veranlassen, von ihrer begeißelten Form in ein unbeweg- liches Palmella-Stadium überzugehen [7]. Hierbei handelte es sich um ein signalgesteuertes Geschehen, bei dem die elektrischen Pulse eine in der Plasmamembran sitzende NADPH-Oxidase aktivieren, die dann reaktive Sauerstoffspezies bildet, die für den Übergang zum Palmella-Stadium wichtig sind Wird die Aktivität dieses Enzyms über einen spezifischen Hemmstoff blockiert, reagieren die Zellen nicht auf die elektrischen Pulse. Der Effekt hat also weniger mit Physik als mit Biologie zu tun.

Wenn kurze elektrische Impulse Signalvorgänge auslösen können, die in Veränderungen der Entwicklung resultieren, dann müsste es eigentlich möglich sein, über eine gezielte Elektroimpulsbehandlung einzelne Gene zu steuern. Um diese Idee zu prüfen, gingen wir zu Haematococcus pluvialis über. Diese einzellige Grünalge ist biotechnologisch interessant, weil sie unter bestimmten Stressbedingungen Astaxanthin bildet, ein rot gefärbtes Carotinoid. Als sehr wirkungsvolles Antioxidans ist Astaxanthin für die Lebensmittelindustrie als Nahrungsergänzungsmittel sehr attraktiv. Durch die oben geschilderten Nanosekundenbehandlung konnten wir tatsächlich drei Schlüsselgene der Astaxanthinsynthese aktivieren (Abb. 1, [8]). Diese Aktivierung beruhte nicht auf einer unspezifischen Stressreaktion der Zelle. Vielmehr konnten wir zeigen, dass diese Impulse trotz eines eigentlich sehr kleinen Energieeintrags eine Signalkette auslösten (Abb. 2). Als früher Schritt wird dabei ein Calciumeinstrom aktiviert. Dieser kann durch geringe Mengen von Gadolinium gehemmt werden. Das deutet darauf hin, dass nicht die elektrisch erzeug- 


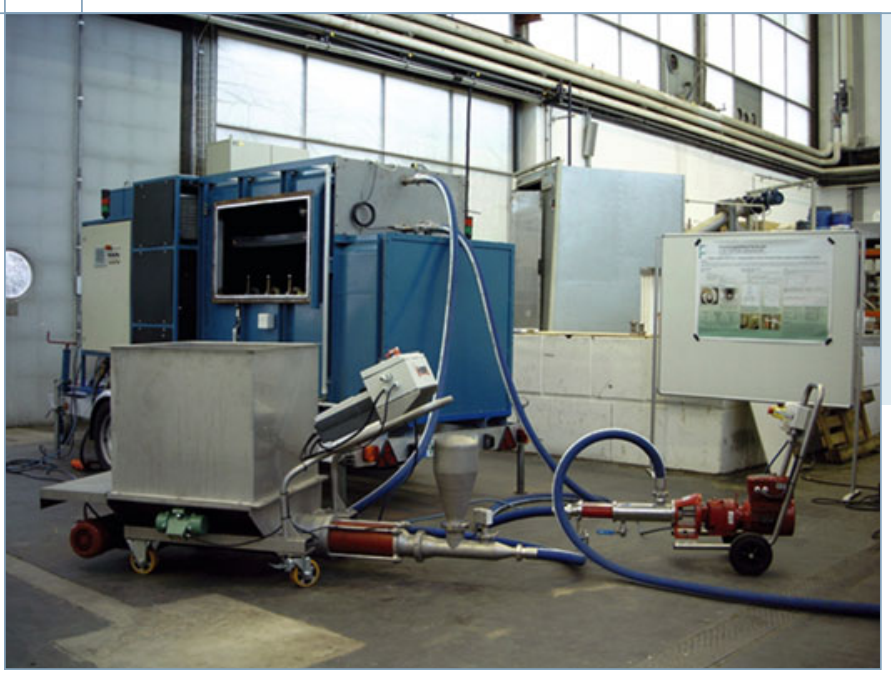

ten Poren in der Membran für diesen Einstrom verantwortlich sind, sondern Calciumkanäle, die bei Pflanzen an der frühen Verarbeitung zahlreicher Signale beteiligt sind.

\section{Nützlicher Selbstmord - biotechnologische Nutzung des elektrisch programmierten Zelltods}

Da es offenbar möglich ist, zelluläre Signalvorgänge über die Elektroimpulsbehandlung gezielt zu steuern, stellte sich die Frage, ob man darüber auch Inhaltsstoffe effizienter und energieschonender gewinnen kann. Anstatt die Zellen auf rein physikalische Weise zu öffnen, könnte man sie vielleicht über die Aktivierung biologischer Signalketten dazu bringen, dies von selbst zu tun. Der durch Signale gesteuerte zelluläre Selbstmord (Apoptose) ist bei tierischen Zellen schon lange bekannt und wird auch medizinisch genutzt, um beispielsweise Tumoren zu therapieren. Vor allem bei Melanomen hat man hier mit Nanosekundenpulsen beeindruckende Erfolge erzielt [9]. Auch bei Pflanzen gibt es einen programmierten Zelltod, der vor allem bei der Abwehr von Krankheitserregern, aber auch während der normalen Entwicklung (etwa bei der Bildung von Leitungsbahnen) wichtig ist. Bis vor kurzem wurde dieser programmierte Zelltod nur bei vielzelligen Pflanzen verortet, weil man davon ausging, dass Einzeller ja miteinander in Konkurrenz stehen, sodass eine Selbstaufopferung zugunsten der anderen Zellen keinen Sinn ergeben würde. Inzwischen hat man jedoch zahlreiche Beispiele gefunden, dass auch einzellige Organismen kooperatives Verhalten zeigen, vor allem unter Stressbedingungen. In der Tat findet man auch bei Algen Metacaspasen - Enzyme, die bei der Exekution des programmierten Zelltods eine zentrale Rolle spielen [10]. Die biologische Funktion scheint darin zu bestehen, dass sich ältere Zellen unter Stressbedingungen nach einem streng geregelten Programm abbauen, um die dadurch das Überleben der gesamten Population verbessert wird.

Diese Idee scheint zu funktionieren. Wenn man den Zellen Zeit gibt, in Antwort auf eine Elektroimpulsbehandlung den programmierten Zelltod einzuleiten, kann man mit deutlich geringerem Energieeintrag eine gute Ausbeute von Inhaltsstoffen erzielen. Beispielsweise führt die Integration einer Inkubationszeit von maximal 24 Stunden dazu, dass man aus Auxenochlorella protothecoides mit einem um den Faktor sechs verringerten Energieaufwand Lipide extrahieren kann [5]. Bei Chlorella vulgaris kann man durch eine Elektroimpulsbehandlung die Bildung von DNS-Leitern auslösen, ein charakteristisches Merkmal für den programmierten Zelltod [11]. Auf der Grundlage dieses Phänomens gelang es inzwischen, die für die Proteinextraktion notwendige Energiemenge für den Zellaufschluss um den Faktor 100 abzusenken [12].

Wie man diese über elektrische Impulse ausgelösten Reaktionen der Zelle dafür nutzen kann, kommerzielle biotechnologische Prozesse effizienter zu machen, steht nun im Fokus der laufenden Forschung (Abb. 3).

\section{Literatur}

[1] Kotnik T, Frey W, Sack M et al. (2015) Electroporationbased applications in biotechnology. Trends Biotechnol 33: 480-488

[2] Sack M, Sigler J, Frenzel S et al. (2010) Research on industrial-scale electroporation devices fostering the extraction of substances from biological tissue. Food Eng Rev 2: 147-156

[3] Akaberi S, Krust D, Müller G et al. (2020) Impact of incubation conditions on protein and C-Phycocyanin recovery from Arthrospira platensis post- pulsed electric field treatment. Bioresour Technol 306: 123099

[4] Goettel M, Eing C, Gusbeth C et al. (2013) Pulsed electric field assisted extraction of intracellular valuables from microalgae. Algal Res 2: 401-408

[5] Silve A, Papachristou I, Wüstner R et al. (2018) Extraction of lipids from wet microalga Auxenochlorella protothecoides using pulsed electric field treatment and ethanol-hexane blends. Algal Res 29: 212-222

[6] Papachristou I, Akaberi S, Silve A et al. (2021) Analysis of the lipid extraction performance in a cascade process for Scenedesmus almeriensis biorefinery. Biotechnol Biofuels 14 $1-14$
[7] Bai F, Gusbeth C, Frey W, Nick P (2017) Nanosecond pulsed electric fields trigger cell differentiation in

Chlamydomonas reinhardtii. BBA Membranes 1859: 651-661 [8] Bai F, Gusbeth C, Frey W, Nick P (2020) Nanosecond pulsed electric fields manipulates modulate the transcription expression of the astaxanthin biosynthesis genes psy, crtR-b and $b k t 1$ during the astaxanthin biosynthesis in Haematococcus pluvialis. Nat Sci Rep 10: 15508 [9] Nuccitelli R, McDaniel A, Anand S et al. (2017) Nanopulse stimulation is a physical modality that can trigger immunogenic tumor cell death. J Immunother Cancer 5: 32 [10] Klemenčič M, Funk C (2018) Structural and functional diversity of caspase homologues in non-metazoan organisms. Protoplasma 255: 387-397

[11] Scherer D, Gusbeth C, Müller G et al. (2019) PEFassisted protein recovery from Chlorella vulgaris is mediated by an enzymatic process after cell death. Algal Res 41: 101536

[12] Krust D, Gusbeth C, Frey W et al. (2022) Biological signalling supports bio-technology - pulsed electric fields specifically release a cell death inducing factor in Chlorella vulgaris. Bioelectrochemistry 143: 107991

Funding note: Open Access funding enabled and organized by Projekt DEAL. Open Access: Dieser Artikel wird unter der Creative Commons Namensnennung 4.0 International Lizenz veröffentlicht, welche die Nutzung, Vervielfältigung, Bearbeitung, Verbreitung und Wiedergabe in jeglichem Medium und Forma erlaubt, sofern Sie den/die ursprünglichen Autor(en) und die Quelle ordnungsgemäß nennen, einen Link zur Creative Commons Lizenz beifügen und angeben, ob Änderungen vorgenommen wurden. Die in diesem Artikel
enthaltenen Bilder und sonstiges Drittmaterial unterliegen ebenfalls der genannten Creative Commons Lizenz, sofern sich aus der Abbildungslegend genannten Creative Commons Lizenz, sofern sich aus der Abbildungsle
nichts anderes ergibt. Sofern das betreffende Material nicht unter der genannten Creative Commons Lizenz steht und die betreffende Handlung nicht nach gesetzlichen Vorschriften erlaubt ist, ist für die oben aufgeführten Weiterverwendungen des Materials die Einwilligung des jeweiligen Rechteinhabers einzuholen. Weitere Details zur Lizenz entnehmen Sie bitte der Lizenzinformation auf http://creativecommons.org/licenses/by/4.0/deed.de.

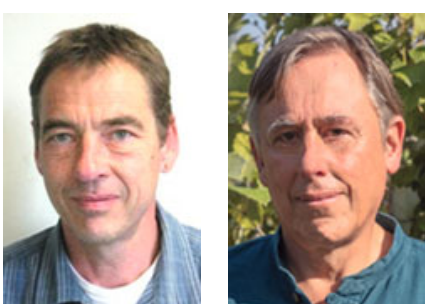

Wolfgang Frey (links) und Peter Nick

Korrespondenzadressen:

Dr. Wolfgang Frey

Karlsruher Institut für Technologie

Institut für Hochleistungsimpuls- und Mikrowellentechnik

Hermann-von-Helmholtz-Platz 1

D-76344 Eggenstein-Leopoldshafen

wolfgang.frey@kit.edu

Prof. Dr. Peter Nick

Karlsruher Institut für Technologie

Botanisches Institut, Molekulare Zellbiologie

Kaiserstraße 2

D-76133 Karlsruhe

peter.nick@kit.edu 